\title{
Enhancement of Neuroplastic P2 and N1c Auditory Evoked Potentials in Musicians
}

\author{
Antoine Shahin, ${ }^{1}$ Daniel J. Bosnyak, ${ }^{2}$ Laurel J. Trainor, ${ }^{2}$ and Larry E. Roberts ${ }^{2}$ \\ ${ }^{1}$ Unit of Medical Physics and Applied Radiation Sciences, and ${ }^{2}$ Department of Psychology, McMaster University, Hamilton, Ontario, Canada L8S 4K1
}

\begin{abstract}
P2 and N1c components of the auditory evoked potential (AEP) have been shown to be sensitive to remodeling of the auditory cortex by training at pitch discrimination in nonmusician subjects. Here, we investigated whether these neuroplastic components of the AEP are enhanced in musicians in accordance with their musical training histories. Highly skilled violinists and pianists and nonmusician controls listened under conditions of passive attention to violin tones, piano tones, and pure tones matched in fundamental frequency to the musical tones. Compared with nonmusician controls, both musician groups evidenced larger N1c (latency, $138 \mathrm{msec}$ ) and P2 (latency, $185 \mathrm{msec}$ ) responses to the three types of tonal stimuli. As in training studies with nonmusicians, N1c enhancement was expressed preferentially in the right hemisphere, where auditory neurons may be specialized for processing of spectral pitch. Equivalent current dipoles fitted to the N1C and P2 field patterns localized to spatially differentiable regions of the secondary auditory cortex, in agreement with previous findings. These results suggest that the tuning properties of neurons are modified in distributed regions of the auditory cortex in accordance with the acoustic training history (musical- or laboratory-based) of the subject. Enhanced P2 and N1c responses in musicians need not be considered genetic or prenatal markers for musical skill.
\end{abstract}

Key words: neural plasticity; musical training; auditory evoked potentials; source analysis; secondary auditory cortex; nature/nurture

\section{Introduction}

Evidence is accumulating that the brains of musicians and nonmusicians respond differently to auditory and tactile stimuli associated with musical performance. Auditory cortical representations evoked by musical tones and measured by the neuromagnetic N1m (latency, $\sim 100 \mathrm{msec}$ ) have been found to be larger in highly skilled musicians than in nonmusicians (Pantev et al., 1998), particularly for musical timbres of the instrument of practice (Pantev et al., 2001). Musical skill is also associated with (1) increased sensitivity of the P300 event-related potential to disparities in melodic contour and pitch interval (Trainor et al., 1999), (2) enhanced neuromagnetic responses evoked by tactile stimulation of the fingering digits of violinists (Elbert et al., 1995), and (3) structural enlargement of the anterior corpus callosum (Schlaug et al., 1995a), the right-sided planum temporale (Schlaug et al., 1995b), and the anteriomedial region of Heschl's gyrus (Schneider et al., 2002) in musicians compared with nonmusicians. Enlargement of Heschl's gyrus in musicians is accompanied by enhanced N19m-P30m middle-latency responses whose cortical generators localize to this region of primary auditory cortex (Schneider et al., 2002). These and other brain attributes associated with musical skill could be determined by prenatal factors or by a genetic code whose expression guides the

Received Dec. 9, 2002; revised April 14, 2003; accepted April 14, 2003.

This research was supported by grants from the Canadian Institutes of Health Research and the Natural Sciences and Engineering Research Council of Canada. We thank Boris Brott and the National Academy Orchestra for their participation, Ed Kucharski for assistance with data collection, and Christo Pantev for helpful comments on this manuscript.

Correspondence should be addressed to L. E. Roberts, Department of Psychology, McMaster University, 1280 Main Street West, Hamilton, Ontario, Canada L8S 4K1. E-mail: roberts@mcmaster.ca.

Copyright $\odot 2003$ Society for Neuroscience $\quad 0270-6474 / 03 / 235545-08 \$ 15.00 / 0$ decision to train musically (Monaghan et al., 1998). Alternatively, these attributes could arise from neuroplastic mechanisms that modify synaptic connections or neural growth processes so as to reflect sensory inputs that are experienced during musical practice (Buonomano and Merzenich, 1998; Edeline, 1999).

Studies of laboratory-induced auditory cortical remodeling in nonmusician subjects are relevant to the neuroplastic hypothesis. Tremblay et al. (2001) and Atienza et al. (2002) trained nonmusician subjects at acoustic discrimination and observed enhancement of the vertex-positive P2 component of the auditory evoked potential (AEP) with a latency near $200 \mathrm{msec}$. This AEP is also enhanced after cochlear implants in the early congenitally deaf (Purdy et al., 2001). Bosnyak et al. (2002) trained nonmusicians to discriminate small changes in the carrier frequency of $40 \mathrm{~Hz}$ amplitude modulated pure tones. In addition to an augmented $\mathrm{P} 2$, a radially oriented AEP identified as the N1c (latency, $\sim 150$ msec) was found to be enhanced specifically in the right hemisphere. Not all AEP components appear to be as sensitive to neuroplastic remodeling as the P2 and N1c. The electrical N1 was not amplified by laboratory training in the above-mentioned EEG studies, although training effects have been reported for its magnetic counterpart, the N1m (Menning et al., 2000). Bosnyak et al. (2002) found that the $40 \mathrm{~Hz}$ auditory steady-state response (SSR) was resistant to amplitude enhancement by training for pitch discrimination in adult nonmusicians, although the phase of the response was modified. Because the $40 \mathrm{~Hz}$ SSR and N19mP30m middle-latency responses localize to Heschl's gyrus and appear to reflect similar processes, amplitude enhancement of these processes in musicians (Schneider et al., 2002) may be an early experience effect or an intrinsic marker for musical ability.

The experiment reported here compared musicians and non- 


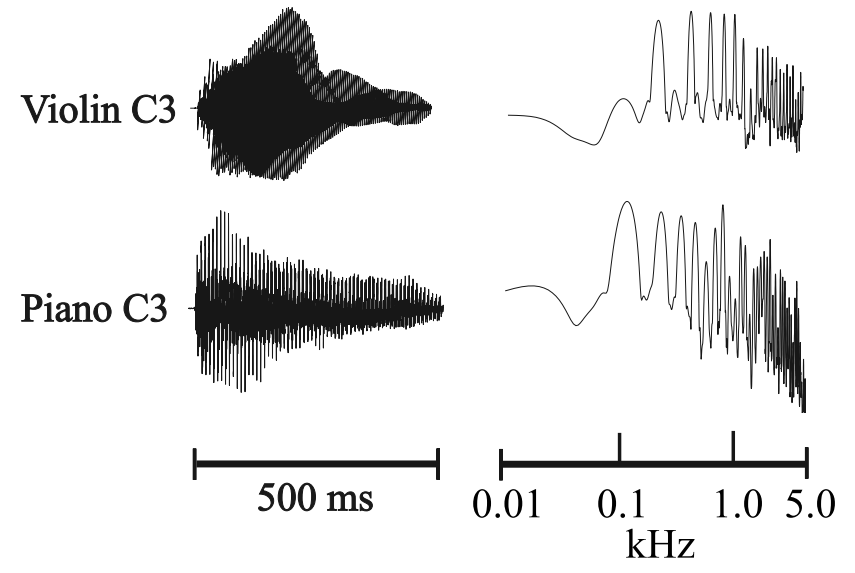

Figure 1. Waveforms (left panel) and spectra (right panel) for the (3 musical stimuli.

musicians for P2 and N1c AEPs that have been found to display neuroplastic properties in training experiments of acoustic discrimination in nonmusician subjects. These responses were expected to be larger in musicians than in nonmusicians when evoked by musical stimuli, in accordance with the sensitivity of the responses to neuroplastic remodeling and the different training histories of these special populations with respect to tones of musical timbre.

\section{Materials and Methods}

Subjects. We investigated 11 professional violinists $(24.3 \pm 2.2$ years of age; six females, five males) who were members of Canada's National Academy Orchestra and nine skilled pianists $(23 \pm 2.5$ years of age; eight females, one male) who had at least grade 10 certification from Canada's Royal Conservatory of Music. Nonmusician controls $(n=14)$ were McMaster University students (22.2 \pm 3.4 years of age; eight females, six males) who did not play a musical instrument and had no formal musical training. Before the experiment, subjects were interviewed to collect information about their musical skills, listening habits, and the musical interests of their parents and siblings. Violinists and pianists had played their instruments for an average of $17 \pm 3.7$ years and $16.6 \pm 4.0$ years, respectively, and practiced for $34.7 \pm 20.8$ and $17.9 \pm 11.1 \mathrm{hr} /$ week, respectively. Nonmusician controls listened passively to music for no more than an average of $0.9 \pm 0.8 \mathrm{hr} /$ day. All subjects except one pianist and one control stated that they were right-handed, and none reported having absolute pitch. Auditory thresholds in the normal range $(<25 \mathrm{~dB}$ at $0.25-8.0 \mathrm{kHz}$ ) were confirmed for each subject by a staircase procedure. Subjects gave written informed consent in accordance with procedures approved by the Research Ethics Committee of McMaster University and were paid $\$ 25$ per hour for their participation.

Procedure. Testing was performed in a sound-attenuated and electrically shielded room. Subjects were presented with violin and piano tones (A3 and C3, American notation) and pure tones matched in fundamental frequency to the musical tones. Each of the six tones was presented 120 times ( 720 tones overall) in a single experimental session that lasted $\sim 45$ min. Tones (500 msec duration, $70 \mathrm{~dB}$ sound pressure level) were delivered monaurally in a mixed order through a speaker placed at eye level $1 \mathrm{~m}$ in front of the subject using a constant stimulus onset asynchrony of $3 \mathrm{sec}$. A passive listening protocol was followed in which subjects read a magazine or newspaper while the tones were presented. The violin tones were taken from Kyma software and pitched to the required notes A3 and C3. The piano tones were obtained from a Korg 01W synthesizer. Pure tones were created with a cosine envelope and matched to the fundamental frequency of the musical tones (see Fig. 1 for the waveforms and spectra of the C3 musical stimuli). The stimuli were judged to be of equal perceived intensity by psychophysical measurements taken from an independent group of nonmusician subjects $(n=10)$ before the experiment.

Electrophysiological recording and analysis. The EEG (32 channels,
$10-20$ system) was sampled at $1 \mathrm{kHz}$ (direct current to $100 \mathrm{~Hz}$, SynAmps; Neuroscan, El Paso, TX) using a $\mathrm{C}_{\mathrm{z}}$ reference and a ground at $\mathrm{AF}_{\mathrm{z}}$. Electrode impedance was reduced to $<10 \mathrm{k} \Omega$ by Electrogel conductant. The continuous EEG file for each subject was digitally filtered at $0.1-20 \mathrm{~Hz}$ (zero phase shift; Scan software version 4.0) and epoched according to stimulus type into $600 \mathrm{msec}$ segments, including a $100 \mathrm{msec}$ prestimulus baseline. Trials containing shifts of $\pm 200 \mu \mathrm{V}$ or greater in any channel were rejected (mean acceptance rate was $86.1 \%$ overall). Accepted trials were averaged according to stimulus type (pure, violin, or piano) collapsing over $\mathrm{C} 3$ and $\mathrm{A} 3$ tones. Averaged data for each subject were exported to Matlab and re-referenced to an average reference for analysis of peak amplitude and latency.

AEP components were identified in the averaged data for each subject and stimulus condition by Matlab algorithms that searched for peak reversals within latency windows determined from group averages. The $\mathrm{N} 1$ peak was determined as the most negative voltage reversal occurring at $\mathrm{C}_{\mathrm{z}}$ during the interval $75-125 \mathrm{msec}$ after stimulus onset. The $\mathrm{P} 2$ peak was determined as the most positive voltage reversal at $C_{z}$ between 140 and $225 \mathrm{msec}$ after stimulus onset. The N1c peak was determined separately at $T_{7}$ and $T_{8}$ as the most negative reversal occurring in the latency window 125-165 msec after stimulus onset. Spherical spline maps of current source density were generated for each AEP component from group averages using Brain Electrical Source Analysis software (BESA 2000) to show scalp topography.

Source analysis of the average-referenced AEP field patterns was performed using BESA. As described in Results, these analyses were conducted separately for each tone and group using the group-averaged data. Because two principal components (one in each hemisphere) accounted for $\geq 96 \%$ of the variance of each of the N1, N1c, and P2 field patterns, two regional sources were used to describe the cortical generators for each AEP (one source in each hemisphere). Regional sources describe cortical activations in terms of three orthogonally related vectors to maximize goodness of fit with the measured field patterns. Regional sources were determined separately for each group and tone at the peaks of each AEP component in the averaged data. Goodness of fit was $>90 \%$ for each source localization (average $93.9 \pm 3.5 \%$ overall).

Statistical analyses. Effects of group (violinists, pianists, and controls) and stimulus (pure tone, violin tone, and piano tone) were evaluated by repeated-measures ANOVA applied to AEPs measured at their amplitude. The variable hemisphere was included when evaluating the N1c and when evaluating differences among the AEPs in the spatial coordinates of their cortical sources. Preplanned group comparisons were made by $t$ tests, and contrasts within the ANOVAs were made by the least significant difference test. All probabilities are two-tailed unless otherwise stated.

\section{Results}

\section{Grand average waveforms}

Figure $2 \mathrm{~A}$ depicts the EEG waveforms evoked by musical tones (violin and piano tones combined) in the nonmusician, violinist, and pianist groups. EEG traces are shown for 28 channels, which were artifact-free and available for analysis in every subject. Two prominent dipolar responses were seen in the two musician groups and the nonmusician controls, labeled N1 and P2, in the violinists' data. $\mathrm{N} 1$ and $\mathrm{P} 2$ components reached their amplitude at the $\mathrm{C}_{\mathrm{z}}$ electrode, with latencies of 97 and $185 \mathrm{msec}$, respectively (groups combined). A third AEP component was detected in the two musician groups and is labeled N1c in the violinists' data in accordance with the nomenclature of Näätänen and Picton (1987). This component reached its amplitude in the right hemisphere ( $\mathrm{T}_{8}$ electrode) at $138 \mathrm{msec}$ between the N1 and P2 responses. Scalp topographies (current source density) are shown for each component at their respective amplitude in Figure $2 B$, averaged over the two musical stimuli in the two musician groups.

Inspection of the waveforms of Figure $2 \mathrm{~A}$ shows that larger P2 responses were evoked by the musical tones in the violinist and 
A $\begin{gathered}\text { Non- } \\ \text { Musicians }\end{gathered}$

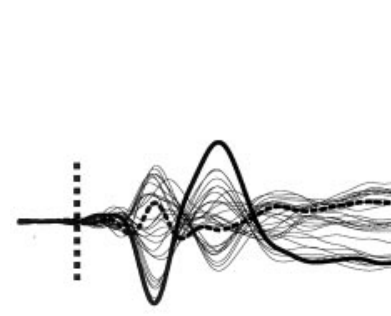

$4 \mu \mathrm{V} \bigsqcup_{100 \mathrm{~ms}}^{\bigsqcup}$

B
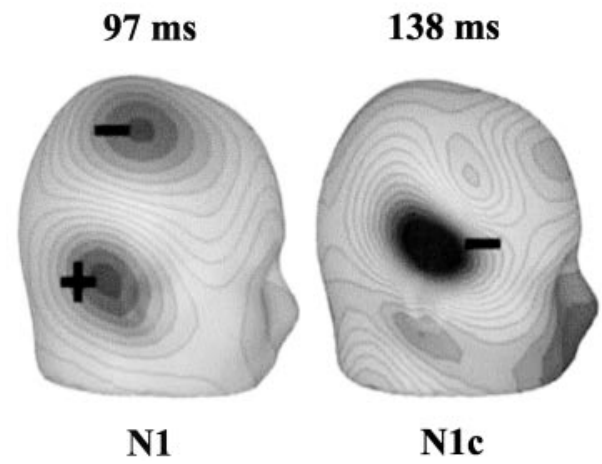

N1c
Pianists

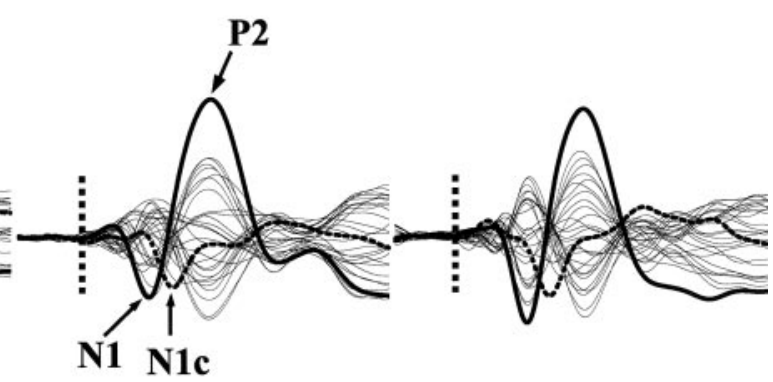

$\mathrm{Cz}$

T8

Violinists

0.0001). Figure 3 also shows that N1 responses elicited by the piano tones were enhanced with respect to the pure tones in all three groups, whereas violin tones evoked smaller $\mathrm{N} 1$ responses than did pure tones in the two musician groups. All contrasts among the three stimuli reached post hoc significance (minimum $p=$ 0.043 , pure vs violin). Although N1 responses to the violin tones were diminished with respect to those of pure tones only in the two musician groups, contrasts comparing N1 responses to the violin tones across groups were not significant.

Effects of group and stimulus on P2 amplitude are shown in the middle panel of Figure 3. Larger P2 responses were observed in violinists and pianists than in nonmusician controls (main effect of group: $\left.F_{(2,31)}=11.36 ; p<0.0001\right)$, whereas the $\mathrm{P} 2$ evoked by violin and piano tones was larger than that evoked by pure tones within each group (main effect of stimulus: $\left.F_{(2,62)}=12.06 ; p<0.0001\right)$. There was no interaction between these variables $\left(F_{(4,62)}=1.07\right)$. The main effect of stimulus was attributable to a larger P2 occurring to the violin tones and to the piano tones than to the pure tones within each group ( $p=0.0001$ or better; post hoc tests), whereas $\mathrm{P} 2$ did not differ between the violin and piano tones within any group. When the violin and piano tones were combined, preplanned contrasts found that larger P2 responses were evoked by the musical stimuli in violinists $\left(t_{(23)}=4.08 ; p=0.0005\right)$ and pianists $\left(t_{(21)}=2.49 ; p=0.02\right)$ compared with nonmusician controls. $\mathrm{P} 2$ responses to the

Figure 2. A, EEG traces (28 channels) evoked by the musical stimuli (violin and piano tones averaged together) are shown separately for the nonmusician, violinist, and pianist groups. The $C_{z}$ electrode is shown in bold, and the $\mathrm{T}_{8}$ electrode is shown as a dotted line. N1, N1c, and P2 responses are identified in the violinists' data. Tone onset is indicated by a dotted vertical line. B, Scalp topography (current source density) and response latency for the three AEP components are shown at their amplitude maxima (the N1c in the right hemisphere). These data were averaged over the two musical stimuli and the two musician groups.

pianist groups compared with nonmusician controls. N1c responses also appeared to be enhanced in the two musician groups. N1 responses, in contrast, did not differ markedly among the three groups. These observations from the averaged data were also evaluated for each AEP component and stimulus in the violinist, pianist, and nonmusician groups.

\section{Analysis of transient responses}

$\mathrm{N} 1$ and $\mathrm{P} 2$ responses were detected at $\mathrm{C}_{\mathrm{z}}$ for all subjects and stimulus conditions, and N1c responses were detected in $86.3 \%$ of 204 possible cases at electrodes $\mathrm{T}_{7}$ and $\mathrm{T}_{8}(34$ subjects $\times$ three stimuli $\times$ two electrodes), with similar detection rates in the three groups $(88.1,81.8$, and $88.9 \%$ for controls, violinists, and pianists, respectively). The peak amplitude of each component is shown in Figure 3, separately for each group and stimulus. All subjects contributed to the N1c responses depicted for each stimulus, which were recorded as the average between $\mathrm{T}_{7}$ and $\mathrm{T}_{8}$ when detected at both electrodes ( $86.3 \%$ of 34 subjects $\times$ three stimuli) or at one of these electrodes when not.

The top panel of Figure 3 shows that N1 responses evoked by each tone were of similar amplitude in the three groups. An ANOVA using the variables group and stimulus yielded no main effects or interactions involving group (both $F$ values $<1$ ). However, a main effect of stimulus was found $\left(F_{(2,62)}=14.07 ; p<\right.$ pure tones were also larger in violinists $\left(t_{(23)}=3.87 ; p=0.0008\right)$ and in pianists $\left(t_{(21)}=2.88 ; p=0.009\right)$ compared with nonmusician subjects.

The results for N1c amplitude are shown in the bottom panel of Figure 3. Findings for the N1c were similar to those obtained for the P2, although the two responses were of opposite polarity and were expressed at different time points and electrode sites (Fig. 2 B). The N1c was enhanced for all stimuli in the two musician groups compared with nonmusician controls, particularly for the violinists. Main effects were found for group $\left(F_{(2,16)}=\right.$ $4.69 ; p=0.0249)$ and for stimulus $\left(F_{(2,32)}=6.817 ; p=0.0034\right)$, with no other term reaching significance. The main effect of stimulus was attributable to larger N1c responses occurring to the piano tone compared with the violin and pure tones $(p=0.05$ or better; post hoc tests), which did not differ from one another. The group effect was attributable to a larger N1c occurring in violinists than in nonmusician controls ( $p=0.026$; post hoc test), with pianists falling between these two groups but differing significantly from neither. When N1c responses evoked by the violin and piano tones were combined, preplanned contrasts showed that musical tones evoked a larger N1c in violinists than in nonmusicians $\left(t_{(13)}=3.16 ; p=0.007\right)$, whereas the difference between pianists and nonmusicians reached one-tailed significance $\left(t_{(9)}=1.91 ; p=0.04\right)$. Similar results were obtained when N1c 

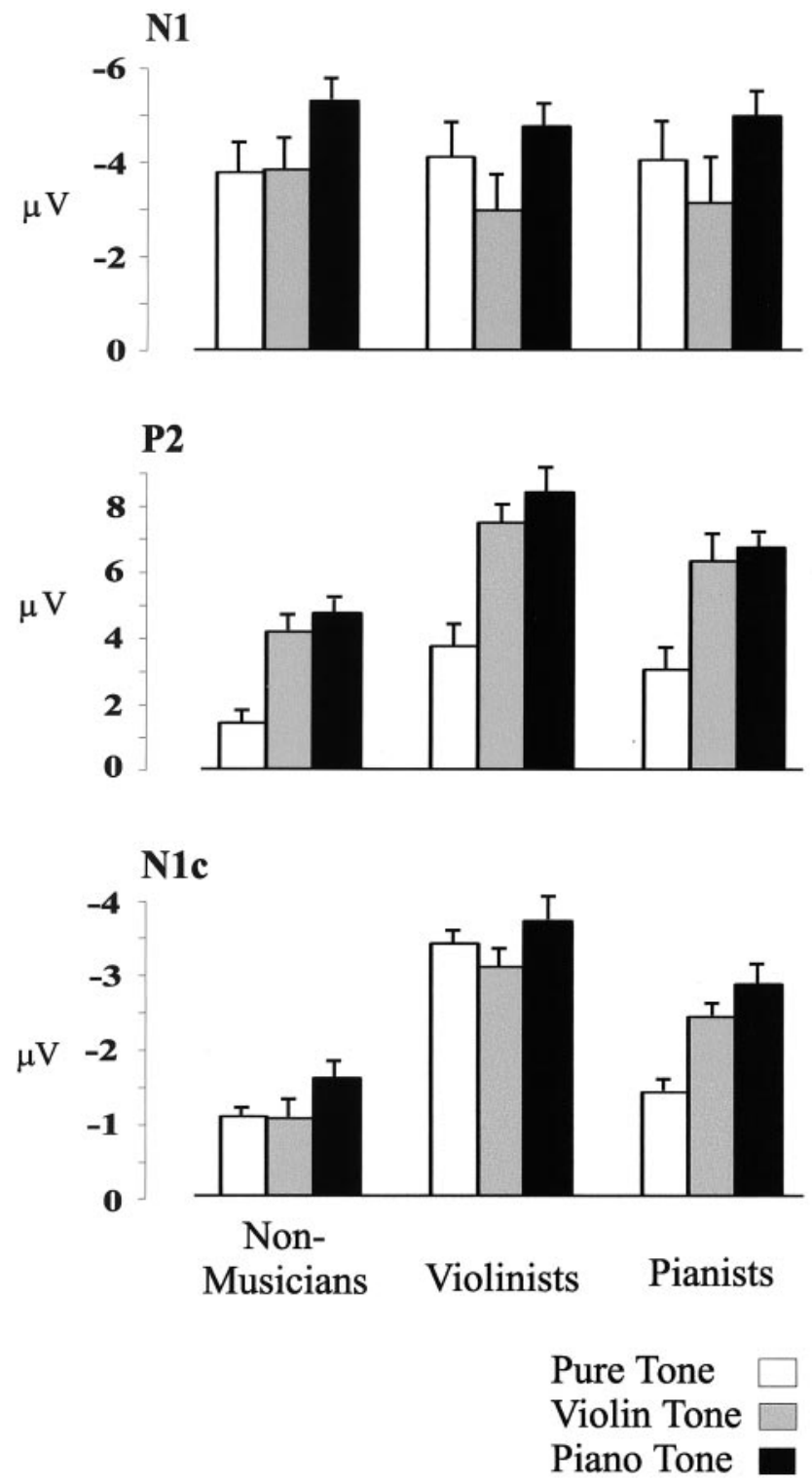

Figure 3. Amplitude of the N1 (top), $\mathrm{P} 2$ (middle), and N1C (bottom) responses is shown separately for each stimulus (pure tone, violin tone, and piano tone) in the nonmusician, violinist, and pianist groups. The bars depict $1 \mathrm{SE}$.

responses evoked by pure tones were contrasted between violinists and nonmusicians $\left(t_{(17)}=3.84 ; p=0.001\right)$ and between pianists and nonmusicians $\left(t_{(17)}=2.05 ; p=0.03\right.$; one-tailed test).

Although hemisphere did not reach significance in the preceding analysis of the $\mathrm{N} 1 \mathrm{c}, \mathrm{N} 1 \mathrm{c}$ responses tended to be larger in the right hemisphere than in the left hemisphere $\left(F_{(1,16)}=3.78\right.$; $p=0.069$ ), and although N1c was detected in $86.3 \%$ of the 204 available cases overall, the majority of detection failures occurred in the left hemisphere [ 20 of $28(71.4 \%) ; p=0.018$; sign test $]$. N1c waveforms recorded from each hemisphere were therefore explored further and are depicted in Figure 4 for each tonal stimulus and group. Inspection of these data showed that N1c in the right hemisphere was enhanced for all stimuli in the two musician groups. N1c responses evoked by violin tones in the right hemisphere were larger in violinists $\left(t_{(18)}=2.357 ; p=0.028\right)$ and in pianists $\left(t_{(18)}=2.535 ; p=0.021\right)$ compared with nonmusician controls, as were N1c responses evoked by piano tones when

\section{Left Hemisphere Right Hemisphere}

Pure

Tone
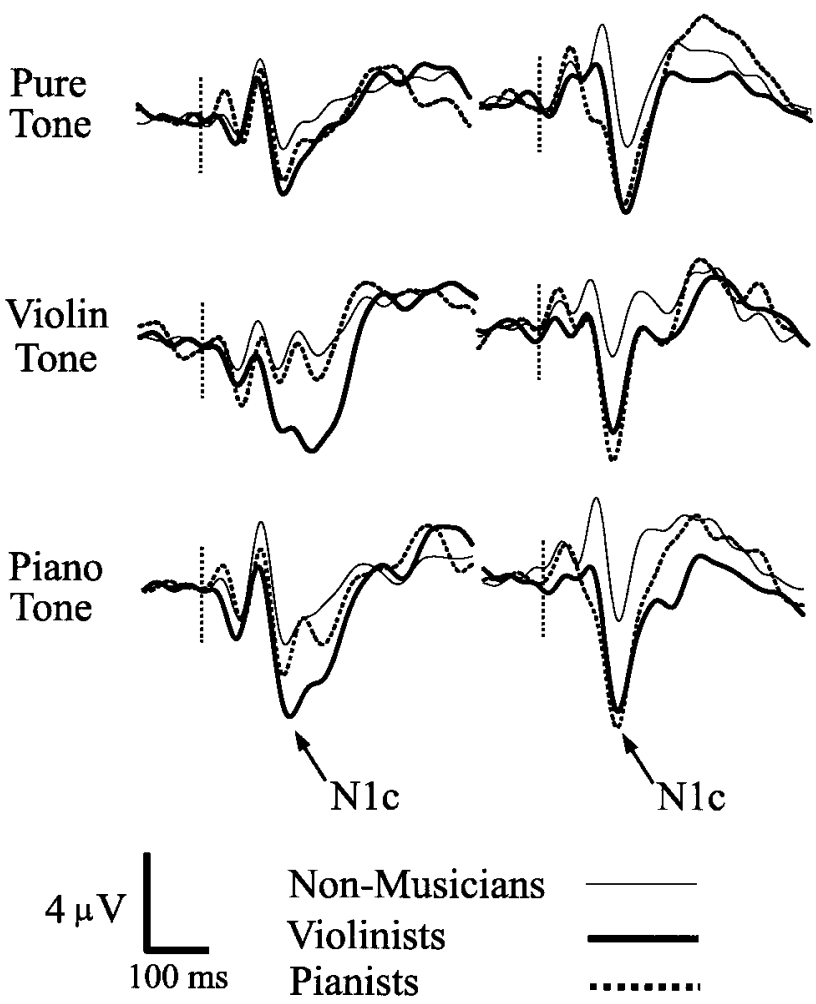

Figure 4. N1c waveforms evoked by the pure, violin, and piano tones are shown for the left and right hemispheres (electrodes $\mathrm{T}_{7}$ and $\mathrm{T}_{8}$, respectively) in the three subject groups. Vertical dotted lines indicate tone onset.

compared between the musician and control groups (violinists: $t_{(23)}=3.18, p=0.004$; pianists: $\left.t_{(20)}=3.035, p=0.006\right) . \mathrm{N} 1 \mathrm{c}$ enhancement was also observed for the pure tone in the right hemisphere when the musician groups were collapsed and compared with nonmusician controls $\left(t_{(29)}=2.17 ; p=0.039\right)$. In the left hemisphere, N1c enhancement approached significance only when violinists were compared with controls $\left(t_{(15)}=2.08 ; p=\right.$ 0.054; musical stimuli combined).

Two subsequent analyses of each AEP were undertaken. The first analysis examined the effect of gender on each AEP component. ANOVAs including the variables gender, AEP, and tone revealed no main effects or interactions attributable to gender when calculated within each group singly or when the three groups were combined (all $F$ values involving gender $<1$ ). The second analysis investigated the effects of group and tone on the latency of each AEP. Main effects attributable to group did not reach significance for any AEP component. However, effects of stimulus were found for the $\mathrm{N} 1\left(F_{(2,62)}=4.63 ; p=0.013\right)$ and the N1c $\left(F_{(2,62)}=81.2 ; p<0.0001\right)$, which were attributable to longer latencies (approximately $+10 \mathrm{msec}$ ) occurring to the violin and piano stimuli than to the pure tones for each AEP. For $\mathrm{N} 1 \mathrm{c}$, an interaction of stimulus with hemisphere was also found $\left(F_{(2,32)}=4.36 ; p=0.021\right)$, which was attributable to faster responses occurring to the musical tones (violin and piano) in the right hemisphere.

\section{Source localizations}

We estimated the centers of cortical activation underlying each AEP component by fitting a regional source to the peak of each 

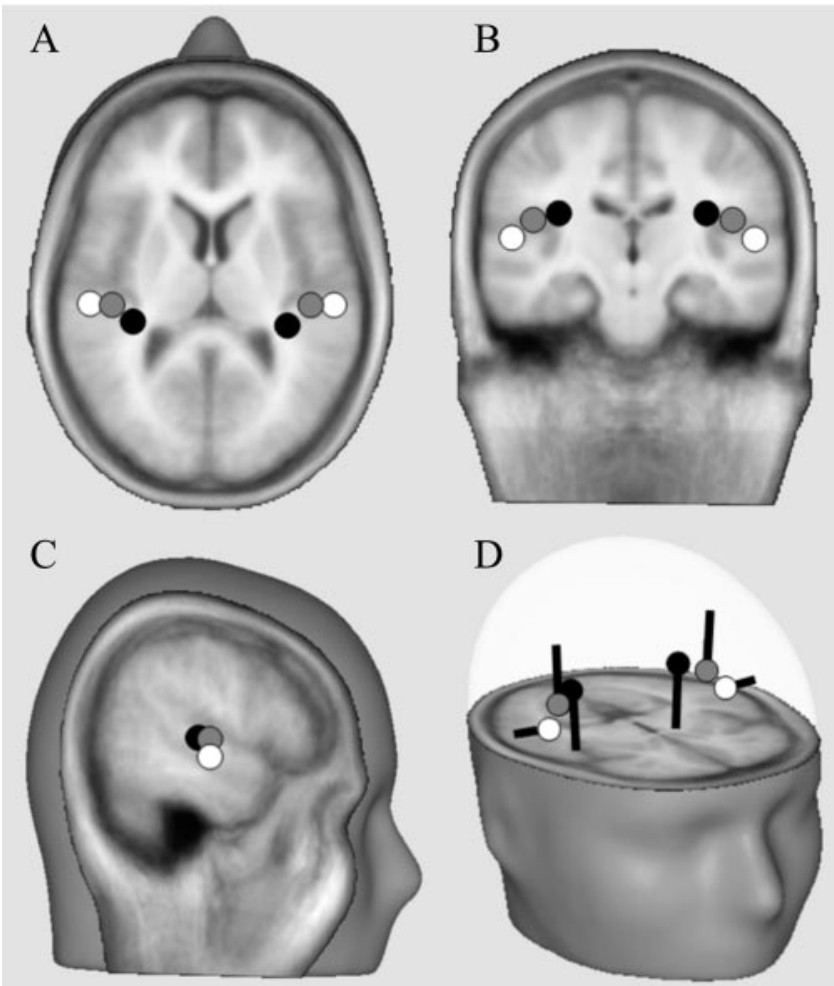

D
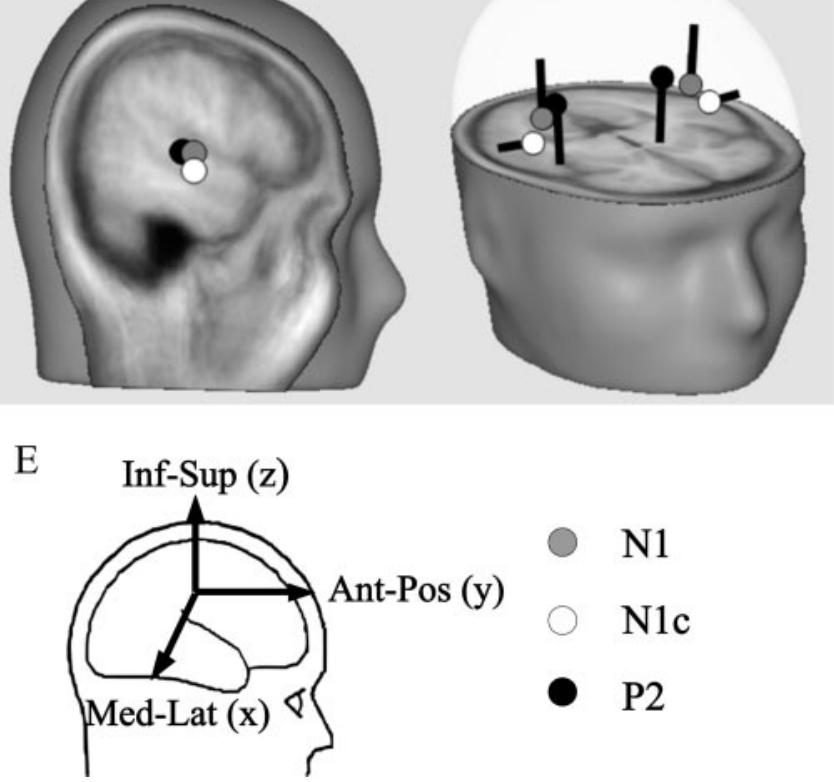

Figure 5. Locations of regional sources determined for the N1, N1C, and P2 are shown in axial $(A)$, coronal $(B)$, and sagittal $(C)$ views superimposed on the average brain of BESA. Source localizations were determined from the grand averaged data and are shown for the condition in which hemispheric symmetry was imposed. $D$, Orientation and strength (dipole moment) of each source are shown in a three-dimensional view (resultant vectors for the $\mathrm{N} 1$ and $\mathrm{P} 2$, radial vector for the N1c, tails negative). Dipole moments are proportional to one another (maximum, $48.6 \mathrm{nAm} ; \mathrm{P} 2$ source, left hemisphere). E, Coordinate system. Ant-P0s, Anteroposterior; Inf-Sup, inferior-superior; Med-Lat, mediolateral.

component using the group-averaged data. Source modeling was conducted in two stages. In stage 1 , two sources (one per hemisphere) were specified, relaxing the constraint that the sources localize symmetrically in the two hemispheres. Spatial coordinates (mediolateral, anteroposterior, and inferior-superior) (Fig. $5 E$ ) for the coordinate system were submitted separately to ANOVAs, collapsing first over stimuli (to examine group effects) and then over groups (to examine stimulus effects). No main effects or interactions involving hemisphere were found, indicating that the relative positions of AEP sources within each hemisphere did not differ between the two auditory cortices of the brain. However, an effect of AEP component was observed in the mediolateral $(x)$ coordinate $\left(F_{(2,12)}=9.87 ; p=0.002\right)$ with P2 sources localizing medial with respect to $\mathrm{N} 1$ and $\mathrm{N} 1 \mathrm{c}$ sources in both hemispheres. A second analysis constrained the cortical sources for each AEP to localize symmetrically in the two hemispheres. Effects attributable to AEP component were again found for the mediolateral coordinate $\left(F_{(2,12)}=21.43 ; p=0.0001\right)$, with P2 sources residing medially and N1c sources residing laterally with respect to $\mathrm{N} 1$ sources ( $p$ ost hoc contrasts: $p<0.02$ or better). An interaction of group with $\operatorname{AEP}\left(F_{(4,12)}=4.76 ; p=0.016\right)$ was also found that was attributable to N1c sources being more lateral in both musician groups compared with nonmusician controls ( post hoc contrasts: $p<0.001$ ). N1c sources tended to reside inferior to $\mathrm{P} 2$ and $\mathrm{N} 1$ generators, although differences in the $z$ coordinate did not reach significance $(p=0.12)$. Regional sources obtained for each AEP are superimposed on the average brain of BESA in Figure 5, in which it can be seen that N1c sources localized to the region of the auditory cortex lateral and inferior to those for the $\mathrm{N} 1$ and $\mathrm{P} 2$, and that P2 sources were medial to those of the N1. No effects of stimulus were found in these analyses, indicating that spatial coordinates for each AEP generator did not depend on this variable.

We also investigated the effect of group, stimulus, and hemisphere on the strength of source activity underlying each AEP component. For this purpose, the lead field matrix of the regional sources determined for each AEP from the grand averaged data was applied to the data of individual subjects. Dipole moment was determined for each subject at the global field maximum of their $\mathrm{N} 1$ and $\mathrm{P} 2$ responses and at the peak of the radial vector for the regional source describing the N1c. Dipole moment averaged over all subjects and conditions is depicted for each AEP in Figure $5 D$ together with dipole orientation (resultant vectors are shown for the N1 and P2). Effects of group and stimulus on dipole moment closely paralleled those obtained for each AEP in Figure 3. A main effect of group was found for the $\mathrm{P} 2\left(F_{(2,31)}=6.43 ; p=\right.$ $0.004)$, which was attributable to larger dipole moments occurring in violinists $(p=0.001)$, but not pianists $(p=0.11)$, compared with nonmusician controls. A main effect of stimulus was also found for P2 $\left(F_{(2,62)}=29.0 ; p=0.0000\right)$, reflecting the fact that larger dipole moments occurred for violin and piano tones compared with pure tones in each group (minimum $p<0.001$; post hoc tests). A main effect of group was obtained for the N1c $\left(F_{(2,31)}=3.91 ; p=0.031\right)$, which reflected a larger N1c occurring in the two musician groups than in controls, especially in the violinists ( $p=0.033$; post hoc test). A larger N1c for piano tones compared with pure tones gave rise to a main effect of stimulus $\left(F_{(2,62)}=7.83 ; p=0.0009\right)$. A main effect of hemisphere was now obtained $\left(F_{(1,31)}=7.706 ; p=0.009\right)$, confirming larger N1c responses occurring on the right side. No effect of group was found on dipole moment for the N1. However, N1 dipole moment differed among the stimuli $\left(F_{(2,62)}=18.2 ; p<0.0001\right)$, with a larger N1 evoked by piano tones and a smaller N1 evoked by violin tones compared with pure tones in each group $(p=0.027$ and 0.005 , respectively; post hoc tests).

\section{Correlations}

The amplitude of the N1, P2, and N1c responses to the musical tones (EEG maxima) and their latencies were correlated with the following questionnaire variables: age of commencement of musical practice, years of practice, hours of weekly practice, and time spent weekly listening to music. Correlations were calculated within the musician groups separately and also when the groups were combined into a single musician sample. No significant correlations were found. This did not change when the correlations were restricted to AEP components evoked by tones of the instrument of practice within the two musician groups. 


\section{Discussion}

Our findings are the first to indicate that P2 and N1c AEPs evoked by musical tones are enhanced in skilled musicians compared with control subjects who have not trained musically. P2 and N1c responses evoked by pure tones that have a pitch-like quality were also enhanced in both musician groups relative to nonmusicians. Enhancement of the N1c and P2 is noteworthy, because these AEPs have been shown in laboratory studies to be sensitive to neuroplastic remodeling when acoustic discriminations are trained in nonmusician subjects (P2; Tremblay et al., 2001; Atienza et al., 2002; Bosnyak et al., 2002) (N1c; Bosnyak et al., 2002). Therefore, our findings corroborate the hypothesis that $P 2$ and N1c enhancements would be expected in musicians compared with nonmusicians, because of the neuroplastic properties of these responses and the different training histories of these groups with respect to tones of musical timbre. It is possible that neuroplastic mechanisms are sufficient to account for enhancement of these AEP components in musicians.

In contrast to the amplitude of the P2 and N1c, the amplitude of the electrical N1 evoked by musical or pure tones did not differ between musicians and nonmusicians in our study. This aspect of our findings concurs with the aforementioned laboratory studies using EEG, which also failed to detect enhanced N1 responses after training for spectral or temporal acoustic discriminations (Tremblay et al., 2001; Atienza et al., 2002; Bosnyak et al., 2002). However, a magnetoencephalography study by Menning et al. (2000) found enhancement of the N1m-evoked field (the magnetic counterpart of the electrical N1) when nonmusicians were trained at pitch discrimination, and the N1m has been reported to be larger in musicians compared with nonmusicians when evoked by piano tones (Pantev et al., 1998). A plausible interpretation of these differing electrical and magnetic findings is that P2 enhancement may commence within the N1 latency window and subtract from its amplitude in electrical recordings, but not in magnetic ones, which are less sensitive to radial currents contributing to the P2 (Jacobson et al., 1991). The N1c, which is generated by radial currents (Woods, 1995; Picton et al., 1999), is also not expressed in magnetic recordings.

In a magnetic study, Pantev et al. (2001) found that the N1m field evoked by trumpet and violin tones in trumpeters and violinists was largest for tones of the instrument of training (timbre specificity). In our study, electrical P2 and N1c responses of similar amplitude were evoked by violin and piano tones in both musician groups. Overlap in the musical training histories of our musicians could have prevented timbre specificity with respect to the N1c and P2. Of our 11 violinists, six reported piano as a secondary instrument, and three of our nine pianists had received previous training on the violin. At present, it is unclear whether enhancement of P2 and N1c responses in musicians compared with nonmusicians relates specifically to musical stimuli and pure tones that possess the musical quality of pitch. Musicians might display larger P2 and N1c responses to acoustic stimuli in general, such as to speech cues that resemble musical stimuli in spectral and temporal complexity, or to noise.

Although the musical background of our subjects did not affect $\mathrm{N} 1$ amplitude, type of stimulus did affect the N1, with larger responses evoked by piano tones than by pure or violin tones in the three groups. The piano tone was characterized by a sharp attack that may have increased the number and/or synchronization of neurons coding for the temporal and spectral features of this stimulus (Schreiner et al., 2000). Spectral complexity may also have contributed to enhancement of $\mathrm{P} 2$ and N1c responses to the two musical stimuli compared with pure tones in each group. Effects of musical background added to these effects of stimulus on the P2 and N1c. Augmented N1c responses (amplitude, dipole moment, detection frequency, and latency) were also more strongly represented in the right hemisphere of our musicians, in agreement with a preferential right-sided expression of N1c reported in previous studies (Woods, 1995; Picton et al., 1999; Bosnyak et al., 2002). Evidence summarized by Zatorre and Belin (2001) indicates that compared with neurons in the left auditory cortex, neurons in the right auditory cortex have higher synaptic densities, more closely spaced cortical columns, and comparatively less myelination, which may reflect a specialization of these neurons for processing of spectral information. It should be noted that our augmented N1c responses did not reflect the opposite end of a current dipole underlying augmented $\mathrm{P} 2$ responses. These two responses reached their amplitude maxima at electrodes oriented orthogonally $\left(\mathrm{C}_{\mathrm{z}}\right.$ and $\left.\mathrm{T}_{8}\right)$, were generated by spatially differentiable cortical sources, and were expressed at latencies differing by $\sim 45 \mathrm{msec}$ in the AEP waveform (Fig. 2).

We evaluated the cortical generators underlying each AEP component by fitting a regional source to the peak of each component. Sources modeled for each component did not differ in spatial location among the tonal stimuli or in their relative positions when compared between the two hemispheres. However, within each hemisphere, the sources of the three AEPs were spatially differentiable in the mediolateral axis, with N1c sources localizing laterally and $\mathrm{P} 2$ sources localizing medially with respect to N1 sources in the region of the superior temporal gyrus (Fig. $5)$. These results are in agreement with other studies comparing N1 and N1c localizations (Scherg et al., 1989; Bosnyak et al., 2002) and with studies comparing N1 with P2 sources (Hari et al., 1987; Joutsiniemi et al., 1989; Pantev et al., 1996) and P2 sources with those of the N1c (Bosnyak et al., 2002). The generators of all three AEP components are centered in the secondary auditory cortex [belt and parabelt regions in the anatomical model of Hackett et al. (2001)] and appear to be differentiable from those of auditory middle-latency and $40 \mathrm{~Hz}$ steady-state responses, which overlap in Heschl's gyrus where the tonotopic maps of primary auditory cortex are found (Liégeois-Chauvel et al., 1993; Pantev et al., 1993; Engelien et al., 2000; Yvert et al., 2001; Bosnyak et al., 2002; Schneider et al., 2002). A constraint on these findings is that source modeling depicts dynamic brain activity at a single point in time and does not preclude multiple generators contributing to each AEP or overlap in their spatial extent. This constraint notwithstanding, spatial differentiation of $\mathrm{P} 2$ and $\mathrm{N} 1 \mathrm{c}$ sources indicates that functional brain activity was altered at distributed sites in the auditory cortex of our musicians compared with subjects who had not trained musically. The greater lateralization of N1c sources in musicians compared with nonmusicians may reflect additional generators contributing to this response in musicians, although the activity of these generators was primarily enhanced in the right hemisphere.

An emerging challenge for neuroscience research is to understand the network behavior underlying remodeling of AEPs by musical and laboratory experience. Physiological evidence gathered from the auditory cortex of the rat (Sukov and Barth, 1998) and from the cat (Mitzdorf, 1985) indicates that negative-going scalp potentials are generated by current sinks occurring on apical dendrites in the superficial neocortical laminas (layers II and III), whereas scalp positivities are produced by depolarization of pyramidal cells in the deeper layers (layers III-VI) consequent on integration of synaptic inputs across the dendritic arbor. If this 
interpretation is accepted for P2 and N1c components of the human AEP, enhancement of the positive-going P2 as well as the negative-going N1c in our musicians is consistent with the interpretation that the number and/or temporal synchrony of neurons representing the trained inputs was augmented by previous musical experience. Animal studies indicate that modulation of the excitability of neocortical neurons by synergistic cholinergic and GABAergic projections from the basal forebrain [nucleus basalis magnocellularis (NBM)] plays an important role in synaptic remodeling (Metherlate and Weinberger, 1990; Freund and Meskenaite, 1992; Buonomano and Merzenich, 1998). Projections from the NBM appear to gate synaptic activity by acting as a teaching signal that strengthens synaptic connections in accordance with Hebbian correlation rules (Cruikshank and Weinberger, 1996; Dykes, 1997; Kilgard and Merzenich, 1998; Kilgard et al., 2001). Modulation by the NBM also has an onset latency resembling that of the auditory N1-P2 complex (Pirch, 1993), as do top-down signals from the prefrontal cortex, which may serve an additional gating function (Tomita et al., 1999). These modulatory influences converging on the sensory scaffold may serve not only a teaching function, but also to amplify communication among neurons whose synapses have been strengthened by training and are receiving input as information is processed in the auditory cortex. This mechanism of learning is consistent with studies of timbre specificity (Pantev et al., 2001) and of training at pitch discrimination (Bosnyak et al., 2002), indicating a degree of spectral specificity in learning, although tuning for the trained stimuli appears to be relatively broad. Cholinergic and GABAergic pathways arising from the basal forebrain project to the sensory cortices in a corticotopic arrangement that would support a broad tuning of synaptic inputs activated by performance on a task (Jiménez-Capdeville et al., 1997).

Our subjects listened to pure, violin, and piano tones while reading a magazine or newspaper. Similar conditions of passive attention have been used in previous studies that have documented functional brain attributes that distinguish musicians from nonmusicians (Elbert et al., 1995; Pantev et al., 1998, 2001; Schneider et al., 2002). This method allows the possibility that enhanced representations could occur in musicians, because musical cues may have a greater command on attention in musically trained subjects than in people who have not trained musically. Consistent with this hypothesis, enhancement of the P2 by attention has been reported (Carpenter et al., 2002), although this effect is variable and may depend on the extent to which the preceding and polarity-opposed N1 is enlarged (cf. Hillyard et al., 1973). Results from acoustic training experiments speak to the role of attention in AEP enhancement. In experiments with humans that cover the electrical P2 (Tremblay et al., 2001; Atienza et al., 2002; Bosnyak et al., 2002), the N1c (Bosnyak et al., 2002), and the magnetic N1m (Menning et al., 2000), progressive enhancements in behavioral performance and in AEPs were observed while subjects attended to the discriminative stimuli to perform the task. Under these conditions, in which attention was actively controlled, changes in behavioral performance and in AEPs were observed that are plausibly attributed to expansion of cortical representations for the trained stimuli. However, modulatory systems in the basal forebrain and prefrontal cortex that may be responsible for attention-like effects are also known to be rapidly conditionable (Pirch et al., 1986; Pirch, 1993). Strengthening of the control of attention by musical cues could therefore be an additional factor, particularly for improvements seen early in training.

\section{References}

Atienza M, Cantero JL, Dominguez-Marin E (2002) The time course of neural changes underlying auditory perceptual learning. Learn Mem 9:138-150.

Bosnyak DJ, Eaton RA, Roberts LE (2002) Enhancement of multiple components of the auditory evoked potential in nonmusicians by training for pitch discrimination with $40-\mathrm{Hz}$ amplitude modulated tones. Proceedings of the 13th International Conference on Biomagnetism, Jena, Germany, August.

Buonomano DV, Merzenich MM (1998) Cortical plasticity: from synapses to maps. Annu Rev Neurosci 21:149-186.

Carpenter M, Cranford JL, Hymel MR, De Chicchis AR, Holbert D (2002) Electrophysiologic signs of attention versus distraction in a binaural listening task. J Clin Neurophysiol 19:55-60.

Cruikshank SJ, Weinberger NM (1996) Receptive-field plasticity in the adult auditory cortex induced by Hebbian covariance. J Neurosci 16:861-875.

Dykes RW (1997) Mechanisms controlling neuronal plasticity in somatosensory cortex. Can J Physiol Pharmacol 75:535-545.

Edeline J (1999) Learning-induced physiological plasticity in the thalamocortical sensory systems: a critical evaluation of receptive field plasticity, map changes and their potential mechanisms. Prog Neurobiol 57:165-224.

Elbert T, Pantev C, Wienbruch C, Rockstroh B, Taub E (1995) Increased cortical representation of the fingers of the left hand in string players. Science 270:305-307.

Engelien A, Schulz M, Ross B, Arolt V, Pantev C (2000) A combined functional in vivo measure for primary and secondary auditory cortices. Hear Res 148:153-160.

Freund TF, Meskenaite V (1992) $\gamma$-Aminobutyric acid-containing basal forebrain neurons innervate inhibitory interneurons in the neocortex. Proc Natl Acad Sci USA 89:738-742.

Hackett TA, Preuss TM, Kaas JH (2001) Architectonic identification of the core region in auditory cortex of macaques, chimpanzees, and humans. J Comp Neurol 441:197-222.

Hari R, Pelizzone M, Makela JP, Hallstrom J, Leinonen L, Lounasmaa OV (1987) Neuromagnetic responses of the human auditory cortex to onand offsets of noise bursts. Audiology 26:31-43.

Hillyard SA, Hink RF, Schwent VL, Picton TW (1973) Electrical signs of selective attention in the human brain. Science 182:177-180.

Jacobson GP, Ahmad BK, Moran J, Newman CW, Tepley N, Wharton J (1991) Auditory evoked cortical magnetic field $\left(\mathrm{M}_{100}-\mathrm{M}_{200}\right)$ measurements in tinnitus and normal groups. Hear Res 56:44-52.

Jiménez-Capdeville ME, Dykes RW, Myasnikov AA (1997) Differential control of cortical activity by the basal forebrain in rats: a role for both cholinergic and inhibitory influences. J Comp Neurol 381:53-67.

Joutsiniemi SL, Hari R, Vilkman V (1989) Cerebral magnetic responses to noise bursts and pauses of different durations. Audiology 28:325-333.

Kilgard MP, Merzenich MM (1998) Cortical map reorganization enabled by nucleus basalis activity. Science 279:1714-1718.

Kilgard MP, Pandya PK, Vazquez J, Gehi A, Schreiner CE, Merzenich MM (2001) Sensory input directs spatial and temporal plasticity in primary auditory cortex. J Neurophysiol 86:326-338.

Liégeois-Chauvel C, Musolino A, Badier JM, Marquis P, Chauvel P (1993) Evoked potentials recorded from the auditory cortex in man: evaluation and topography of the middle latency components. Electroencephalogr Clin Neurophysiol 92:204-214.

Menning H, Roberts LE, Pantev C (2000) Plastic changes in the auditory cortex induced by intensive frequency discrimination training. NeuroReport 11:817-822.

Metherlate R, Weinberger NM (1990) Cholinergic modulation of responses to single tones produces tone-specific receptive field alterations in cat auditory cortex. Synapse 6:133-145.

Mitzdorf U (1985) Current source-density method and application in cat cerebral cortex: investigation of evoked potentials and EEG phenomena. Physiol Rev 65:37-100.

Monaghan P, Metcalfe NB, Ruxton GD (1998) Does practice shape the brain? Nature 394:4343.

Näätänen R, Picton T (1987) The N1 wave of the human electric and magnetic response to sound: a review and analysis of the component structure. Psychophysiology 24:375-425.

Pantev C, Elbert T, Makeig S, Hampson S, Eulitz C, Hoke M (1993) Rela- 
tionship of transient and steady-state auditory evoked fields. Electroencephalogr Clin Neurophysiol 88:389-396.

Pantev C, Eulitz C, Hampson S, Ross B, Roberts LE (1996) The auditory evoked "off" response: sources and comparison with the "on" and the "sustained" responses. Ear Hear 17:255-265.

Pantev C, Oostenveld R, Engelien A, Ross B, Roberts LE, Hoke M (1998) Increased auditory cortical representation in musicians. Nature 392:811-814.

Pantev C, Roberts LE, Schulz M, Engelien A, Ross B (2001) Timbre-specific enhancement of auditory cortical representations in musicians. NeuroReport 12:1-6.

Picton TW, Alain C, Woods DL, John MS, Scherg M, Valdes-Sosa P, BoschBayard J, Trujillo NJ (1999) Intracerebral sources of human auditory evoked potentials. Audiol Neurootol 4:64-79.

Pirch JH (1993) Basal forebrain and frontal cortex neuron responses during visual discrimination in the rat. Brain Res Bull 31:73-83.

Pirch JH, Corbus MJ, Rigdon GC, Lyness WH (1986) Generation of cortical event-related slow potentials in the rat involves nucleus basalis cholinergic innervation. Electroencephalogr Clin Neurophysiol 63:464-475.

Purdy SC, Kelly AS, Thorne PR (2001) Auditory evoked potentials as measures of plasticity in humans. Audiol Neurootol 6:211-215.

Scherg M, Vajsar J, Picton TW (1989) A source analysis of the late human auditory evoked potentials. Cogn Neurosci 1:336-355.

Schlaug G, Jancke L, Huang Y, Staiger JF, Steinmetz H (1995a) Increased corpus callosum size in musicians. Neuropsychologia 33:1047-1055.

Schlaug G, Jäncke L, Huang Y, Steinmetz H (1995b) In vivo evidence of structural brain asymmetry in musicians. Science 267:699-701.
Schneider P, Scherg M, Dosch HG, Specht HJ, Gutschalk A, Rupp A (2002) Morphology of Heschl's gyrus reflects enhanced activation in the auditory cortex of musicians. Nat Neurosci 5:688-694.

Schreiner CE, Read HL, Sutter ML (2000) Modular organization of frequency integration in primary auditory cortex. Annu Rev Neurosci 23:501-529.

Sukov W, Barth DS (1998) Three-dimensional analysis of spontaneous and thalamically evoked gamma oscillations in auditory cortex. J Neurophysiol 79:2875-2884.

Tomita H, Ohbayashi M, Nakahara K, Hasegawa I, Miyashita Y (1999) Topdown signal from prefrontal cortex in executive control of memory retrieval. Nature 401:699-703.

Trainor LJ, Desjardins RN, Rockel C (1999) (1999) A comparison of contour and interval processing in musicians and nonmusicians using eventrelated potentials. Aust J Psychol 51:147-153.

Tremblay K, Kraus N, McGee T, Ponton C, Otis B (2001) Central auditory plasticity: changes in the N1-P2 complex after speech-sound training. Ear Hear 22:79-90.

Woods DL (1995) The component structure of the N1 wave of the human auditory evoked potential. In: Perspectives of event-related potential research. Electroencephalogr Clin Neurophysiol [Suppl] 44:102-109.

Yvert B, Crouzeix A, Bertrand O, Seither-Preisler A, Pantev C (2001) Multiple supratemporal sources of magnetic and electric auditory evoked middle latency components in humans. Cereb Cortex 11:411-423.

Zatorre RJ, Belin P (2001) Spectral and temporal processing in human auditory cortex. Cereb Cortex 11:946-953. 